CANTUA. 2017; 16: 44-53

Fecha de recepción: 24.07.2017

Fecha de aceptación: 18.11.2017

\title{
Comunidad de macroinvertebrados en el río Hatunmayo
}

\section{Macroinvertebrate community in the hatunmayo river}

\section{Luciano J. Cruz- Miranda ${ }^{1, a}$, Cynthya Alegre- Palomino ${ }^{1, b}$}

\author{
${ }^{1}$ Escuela Profesional de Biología, Facultad de Ciencias, Universidad Nacional de San Antonio Abad \\ del Cusco. Cusco,Perú. \\ ${ }^{\mathrm{a}}$ ORCID 000-0002-4407-6184; ${ }^{\mathrm{b}}$ ORCID 0000-0001-9099-2290
}

\section{RESUMEN}

El presente estudio corresponde a los meses de setiembre a diciembre de la estación de primavera del 2014 en las aguas del río Hatunmayo, Anta - Cusco, desde la cabecera de cuenca hasta su desembocadura en el río Vilcanota. Este recurso hídrico en su recorrido recibe afluentes de los ríos Cachimayo y Pucyura, realizando su recorrido por Izcuchaca y se une con el río Pitumayo en Huarocondo, por lo que sufre variaciones en todo el trayecto en sus características física y químicas, además de recibir agua residuales y de otras actividades, por lo que se estimó evaluar la comunidad de macroinvertebrados como bioindicadores en 20 estaciones pre establecidas para determinar la calidad de agua, aplicando la metodología del paquete de hojas. La composición de macroinvertebrados en el río Hatunmayo, está conformado por 13449 individuos, distribuidos en 13 órdenes y 35 familias, predominando los órdenes Amphipoda y Diptera. La diversidad según el índice de Shannon es mediana y según el indice de Simpson es baja y presenta una alta dominancia. Los Índices Biológico de Familia (IBF) y el índice Ephemeroptera, Plecóptera y Tricoptera (EPT), califican al río Hatunmayo con una calidad de agua de mala a regular desde su naciente hasta su desembocadura.

PALABRAS CLAVE: Macroinvertebrados, calidad de agua, paquete de hojas, Índice de diversidad. 


\begin{abstract}
This study corresponds to the months of September to December of the 2014 spring season in the waters of the Hatunmayo River, Anta - Cusco, from the head of the basin to its mouth in the Vilcanota River. This water resource on its way receives tributaries from the Cachimayo and Pucyura rivers, making its journey through Izcuchaca and joins the Pitumayo river in Huarocondo, for which it undergoes variations throughout the journey in its physical and chemical characteristics, in addition to receiving water. residuals and other activities, for which it was estimated to evaluate the macroinvertebrate community as bioindicators in 20 pre-established stations to determine water quality, applying the leaf pack methodology. The composition of macroinvertebrates in the Hatunmayo River is made up of 13449 individuals distributed in 13 orders and 35 families, with the Amphipoda and Diptera orders predominating. The diversity according to the Shannon index is medium and according to the Simpson index it is low and presents a high dominance. The Biological Family Indices (IBF) and the Ephemeroptera, Plecóptera and Tricoptera (EPT) index, qualify the Hatunmayo river with a water quality from poor to regular from its source to its mouth.
\end{abstract}

KEY WORDS: Macroinvertebrates, water quality, leaf pack, diversity index.

\title{
INTRODUCCIÓN
}

Los macroinvertebrados son usados como bioindicadores para determinar la calidad de medios acuáticos que, por sus características, requerimientos especiales y adaptaciones a diferentes condiciones ambientales, los convierte en organismos con límites de tolerancia específicos a diferentes alteraciones de su hábitat. Asimismo, la capacidad natural de respuesta hacia perturbaciones al que son sometidos, se refleja en su composición del medio acuático que habitan y está determinada por la dominancia de las poblaciones de organismos adaptados a características propias de la calidad de sus aguas (Zamorra, 2011).

La variabilidad de las características físicas y químicas, incluyendo el sustrato y la velocidad de la corriente del río, son factores que pueden influenciar en la diversidad biótica de los macroinvertebrados (Roldan 1999).

Las condiciones naturales del río Hatunmayo sufren alteraciones en su recorrido desde su naciente hasta su desembocadura como afluente al río Vilcanota en sus parámetros físicos, químicos y biológicos, debido al crecimiento poblacional que induce a la expansión urbana en diversos tramos de su recorrido, las actividades antrópicas, vertimientos de aguas residuales y residuos sólidos, uso de fertilizantes, insecticidas y pesticidas. Las aguas del río están destinadas al riego de cultivos y bebida de animales domésticos, por tanto, la agricultura y ganadería son principales actividades económicas de las familias ubicadas en la cuenca y es necesario conocer el estado actual de la calidad del río. 
Por estas consideraciones, se desarrolló el presente estudio con el objeto de evaluar la comunidad de macroinvertebrados y determinar el grado de calidad de agua del río Hatunmayo durante el periodo de primavera (setiembre a diciembre) del 2014, así mismo, tener un mayor conocimiento del funcionamiento de este ecosistema dulceacuícola que permitan proponer alternativas de conservación y protección para una gestión sostenible del recurso hídrico en las provincias de Cusco, Anta y Urub

\section{MÉTODOS}

\section{Área de estudio}

El río Hatunmayo nace de la unión de los ríos Querahuaylla y Yanamayo, y hasta su desembocadura se encuentra en las Provincias de Cusco, Anta y Urubamba, entre las coordenadas UTM N: 8518822 8531044 y E: 827287 - 8014230, alcanzando una superficie de $797.67 \mathrm{Km}^{2}$ recorriendo desde los $4672 \mathrm{msnm}$ hasta los $2828 \mathrm{msnm}$ (figura 1).

La cuenca del Hatunmayo se encuentra en las zonas de vida: bosque seco montano bajo subtropical y bosque húmedo montano subtropical con una temperatura promedio anual de $11.17{ }^{\circ} \mathrm{C}$ y una precipitación anual de $808.2 \mathrm{~mm}$.

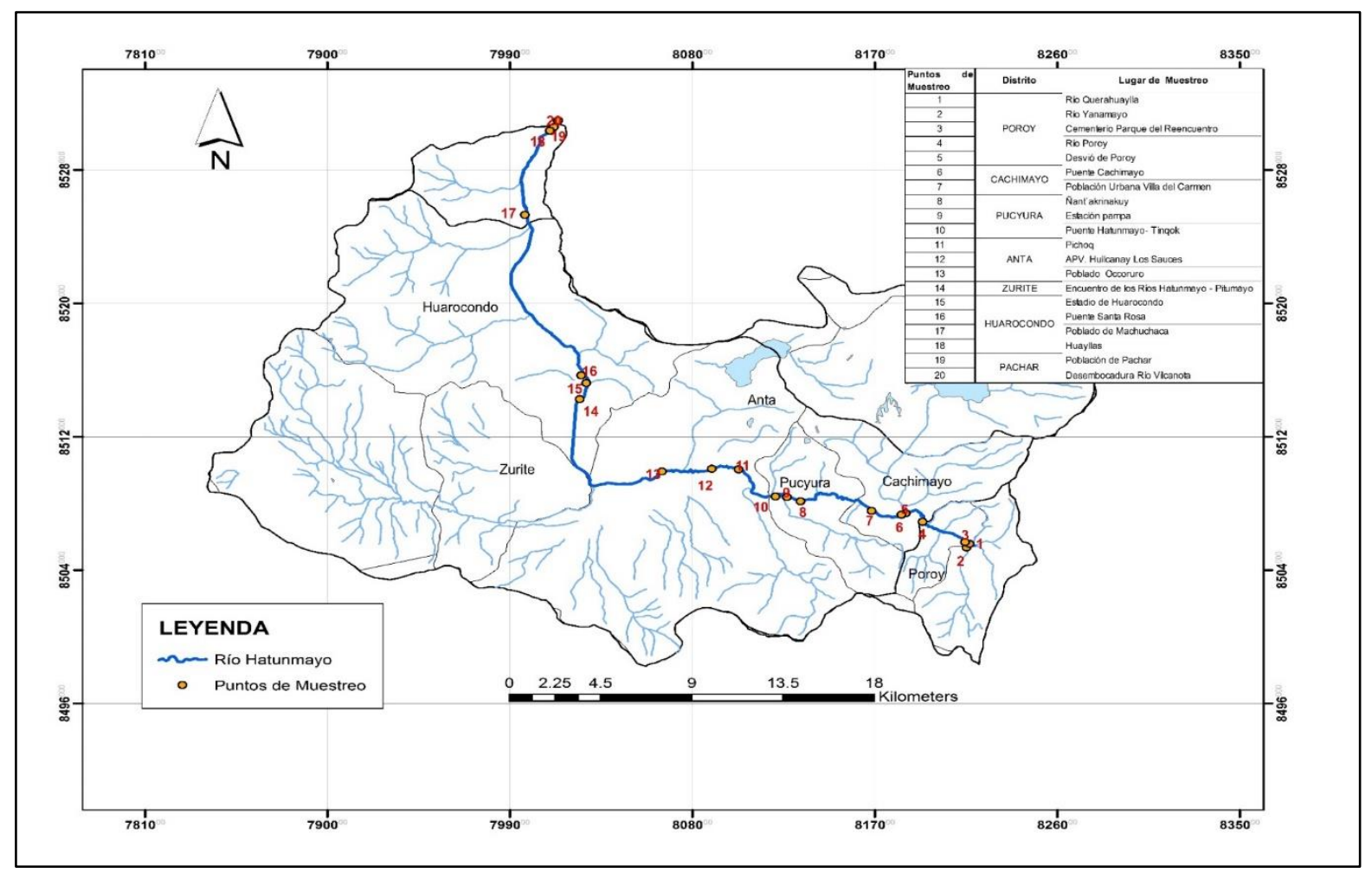

Figura 1. Mapa de ubicación de puntos de muestreo 


\section{Muestreo}

Los puntos de muestreo fueron determinados considerando los siguientes aspectos:

- Poblaciones asentadas en ambas márgenes del río

- Vertimientos de aguas residuales y de residuos sólidos

- Efluente de agua de piscigranjas

- Efluentes de camales (Poroy y Anta)

- Actividades económicas (agricultura, ganadería y extracción de minería no metálica)

Las características de temperatura, oxígeno disuelto y $\mathrm{pH}$ se registraron in situ con un Instrumento multiparamétrico HD 98569 (Método Directo).

El caudal del río se calculó mediante el método velocidad/superficie.

\section{Colección de macroinvertebrados}

Mediante el paquete experimental de "Leaf Pack Experiment kit", denominado también paquete de hojas desarrollado por Stroud Water Research Center, se recolectó hojas secas de arbustos y vegetación circundante al río colocándolo en bolsas de malla plástica con un peso de $30 \mathrm{~g}$ de hojas. Los paquetes se colocaron en un número de 03 por cada punto de muestreo en un rápido del río sujetado por una cuerda a una rama o árbol, considerando que el paquete de hojas este totalmente sumergido y se mantengan intactas durante un periodo de 21 a 31 días.

Posterior a los 21 días de exposición, se extrajo los paquetes de hojas del agua, rápida y suavemente en contra corriente, colocándolos en una bandeja de fondo blanco con agua, posteriormente se separó las hojas y las muestras preservando en alcohol a $75 \%$ debidamente rotulado y etiquetado por cada punto de muestreo para su posterior procesamiento e identificación taxonómica en laboratorio.

Se determinaron los Índices Biológico de Familias (IBF) y el Índice Ephemeroptera, Plecoptera y Tricoptera (EPT), los índices de diversidad de Shannon - Weaver y Simpson.

\section{RESULTADOS}

El caudal del río Querahuaylla es de $0.007 \mathrm{~m}^{3} / \mathrm{seg}$. y del río Yanamayo $0.069 \mathrm{~m}^{3} / \mathrm{seg}$, por consiguiente, el río Hatunmayo inicia su recorrido con un caudal de $0.076 \mathrm{~m}^{3} / \mathrm{seg}$ el mismo que va incrementando su caudal en toda la cuenca por ser el recolector principal; disminuye su caudal en el distrito de Pucyura debido al uso de este recurso hídrico para riego de cultivos de maíz, papa y forraje, desembocando al río Vilcanota con $0.642 \mathrm{~m}^{3} / \mathrm{seg}$. 
La temperatura del agua varía de $12^{\circ} \mathrm{C}$ a $20.6^{\circ} \mathrm{C}$, el $\mathrm{pH}$ se halla en un rango de $7.89-9.13$, con tendencia a la basicidad, el valor máximo se registra en el punto de muestreo de Huayllas probablemente debido al arrastre de materiales calizos por los constantes deslizamientos en dicha zona. La concentración de oxígeno disuelto varía por muchos factores como la fotosíntesis, la respiración, penetración de la luz, materia orgánica, entre otros, con valores de $5.47 \mathrm{mg} / \mathrm{L}$ a 9.58 $\mathrm{mg} / \mathrm{L}$.

\section{Macroinvertebrados}

Durante el periodo de evaluación se colecto un total de 13449 individuos de macroinvertebrados, distribuidos en 35 familias y 15 órdenes que corresponden a los puntos de control y estaciones experimentales; registrándose especies tolerantes a la contaminación como los órdenes Oligochaeta, Gasteropoda, Díptera (familia Simuluiidae, Chironmidae) e Hirudinea, algo tolerantes a la contaminación los órdenes Coleóptera, Odonata y Amphipoda y los órdenes sensibles a la contaminación son Plecoptera, Ephemeroptera y Tricoptera, presentando un menor número de especies (figura 2 y anexo 1 ).

El número de individuos registrados es variable, siendo el orden Amphipoda el más representativo con la familia Hyalellidae, seguido por el orden Diptera con la familia Chironomidae y el orden Ephemeroptera con la familia Baetidae con 3558, 2298 y 1748 individuos respectivamente, el orden Tricoptera registra el menor número de individuos con las familias de Leptoceridae y Ecnomidae representados con solo 1 individuo cada familia.

El índice de diversidad según Shannon, se encuentra entre los rangos de 1.312 a 2.084 considerando una diversidad de poca a mediana y el índice de Simpson, se encuentra entre los rangos de 0.5682 a 0.8322 , con una baja diversidad.

El índice IBF desarrollado por Hilsenhoff en 1988, el río Querahuaylla y el río Yanamayo, presentan valores de 5.781 y 5.445 que denotan una calidad de agua relativamente de mala a regular respectivamente, el río Hatunmayo a la altura del Pitumayo presenta un IBF de 6.84 que denota una calidad de agua mala y en la zona de Huayllas-Huarocondo, la calidad varia de mala a muy buena, probablemente por el proceso de autodepuración y la pendiente que tiene esta zona, desembocando al río Vilcanota con un valor de 5.503 que califica una calidad regular.

La clasificación de la calidad de agua según el índice EPT desarrollado por Leiva M. en 2004, para el río Querahuaylla es de 22 con una calidad de agua mala y el río Yanamayo con 26 de calidad de agua regular. Así mismo, el río Hatunmayo realiza su recorrido por los distritos de Cachimayo, Pucyura, Anta, Zurite y Huarocondo con una calidad de agua de regular a mala desemboca al río Vilcanota con un EPT de 48 con una calidad regular. 


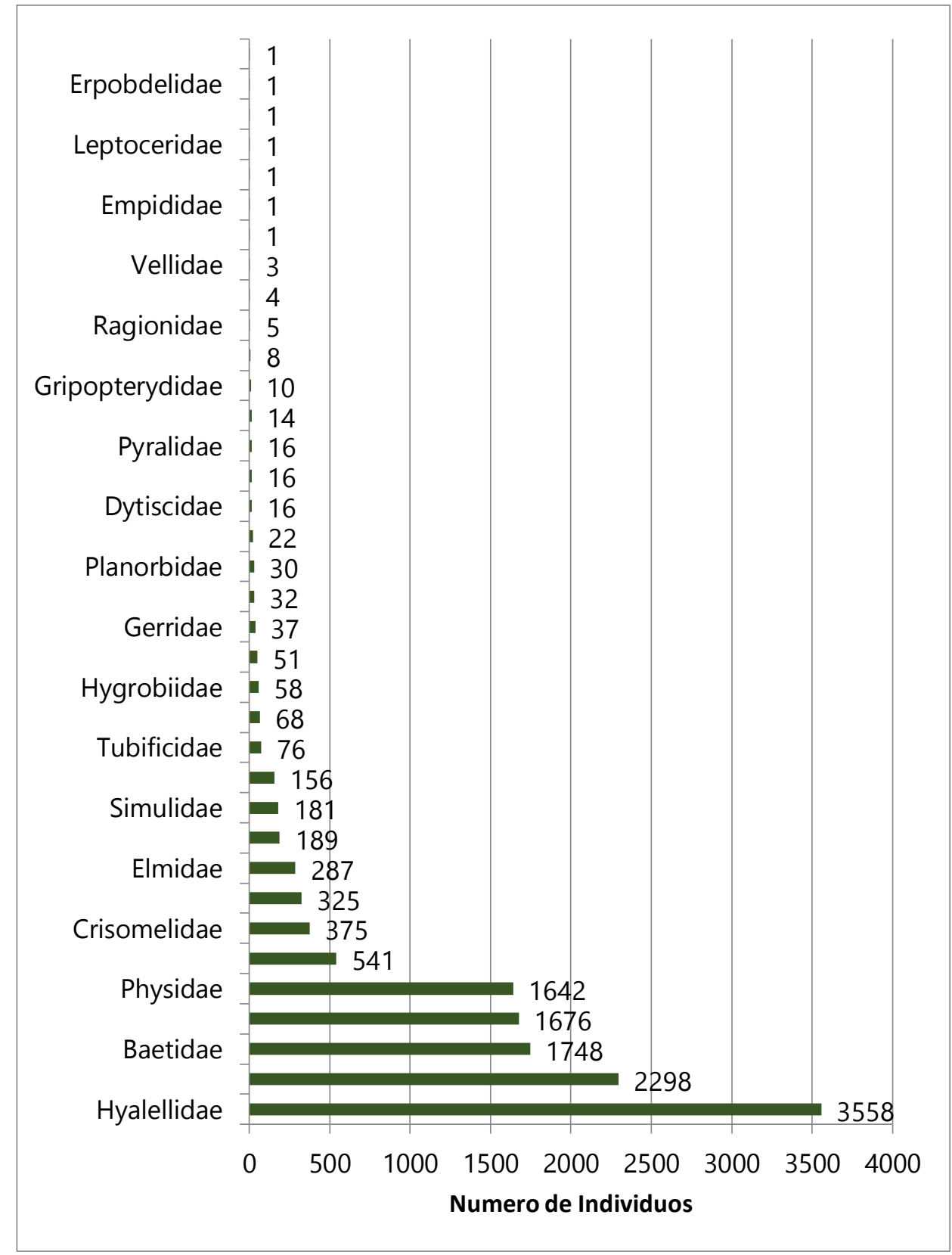

Figura 2. Número de Individuos por familia. 


\section{DISCUSION}

El caudal del río Hatunmayo desde su naciente de la conjunción de los ríos Querahuaylla y Yanamayo, hasta su desembocadura en el río Vilcanota incrementa su caudal en 8.4 veces, lo que influye en la distribución de los macroinvertebrados, además de ser considerado de un régimen irregular. La temperatura del agua varía de $12^{\circ} \mathrm{C}$ a $20.6^{\circ} \mathrm{C}$, en consecuencia, esta influye en la cantidad y diversidad de la vida acuática. Muchos organismos son sensibles a valores extremos de $\mathrm{pH}$ y están ausentes en aguas con $\mathrm{pH}$ por debajo de 4,0; es decir un pequeño cambio en el $\mathrm{pH}$ podría tener efectos significativos en la calidad del agua, según referencia de Stroud Water Research Center (2012). Por tanto, el pH registrado supera los estándares Nacionales de Calidad Ambiental para el Agua (ECA), pertenecientes a la categoría 3: riego de vegetales y bebida de animales.

El oxígeno disuelto es un indicador de la contaminación del agua y de la capacidad para dar soporte a la vida vegetal y animal; además destaca que, un nivel alto de oxígeno disuelto indica agua de mejor calidad y niveles bajos de oxígeno disuelto se pueden encontrar en áreas donde el material orgánico esta en descomposición; por lo que, la concentración de oxígeno registrado varia de $5.47 \mathrm{mg} / \mathrm{L}$ a $9.58 \mathrm{mg} / \mathrm{L}$., este incremento de la concentración se debe a la pendiente del río en la cuenca baja que corresponde de Huarocondo a Pachar, que genera movimientos hidrodinámicos considerables.

La abundancia y riqueza de familias es variable en los puntos de muestreo influenciado directamente por los parámetros físicos y químicos del agua.

Según los Índices Biológico de Familias (IBF) y el Índice Ephemeroptera, Plecoptera y Tricoptera (EPT), la calidad varia de regular a buena; según Alba-Tercedor (1996), los macroinvertebrados son extremadamente sensibles a perturbaciones, por lo que la variación en la composición y estructura de sus comunidades pueden interpretarse como consecuencia de algún agente de contaminación que ingresa al río. Igualmente, Roldan G. (1997) manifiesta que las perturbaciones altas, hacen que desaparezcan las especies intolerantes. 


\section{CONCLUSIONES}

Los parámetros físicos y químicos del río Hatunmayo, (temperatura, oxígeno disuelto) se encuentran dentro de los Estándares Nacionales de Calidad Ambiental para Agua correspondiente a la categoría 3, no así el pH que supera los valores establecidos para esta categoría. La velocidad del río desde su naciente hasta su desembocadura varia de $0.178 \mathrm{~m} / \mathrm{seg}$ a $0.340 \mathrm{~m} / \mathrm{seg}$.

La comunidad de macroinvertebrados del río Hatunmayo está conformado por 35 familias distribuidos en 13 órdenes, donde predomina la familia Hyalellidae, seguido por la familia Chrironomidae.. La diversidad de macroinvertebrados para el río Hatunmayo según el índice de Shannon varía de 1.4 a 2.08 que refleja mediana diversidad, mientras que el índice de Simpson varía de 0.52 a 0.83 con una baja diversidad y alta dominancia. Los Índices Biológico de Familia (IBF) y el índice Ephemeroptera, Plecóptera y Tricoptera (EPT), califican al río Hatunmayo con una calidad de agua de mala a regular desde su naciente hasta su desembocadura.

\section{Autor corresponsal.}

Cynthya Alegre- Palomino

Correo electrónico: cynthyalegre2@gmail.com

\section{REFERENCIAS BIBLIOGRÁFICAS}

Alba, J. (1996). Macroinvertebrados acuáticos y calidad de las aguas de los ríos. IV Simposio del agua en Andalucia..

MINAM (2017). Aprueban los Estándares Nacionales de Calidad Ambiental para Agua. Decreto Supremo No04-2017-MINAM. Diario Oficial El Peruano.

Hanson, P., Springer, M. \& Ramírez, A. (2010). Introducción a los grupos de macroinvertebrados acuáticos. Escuela de Biología, Universidad de Costa Rica.

Hilsenhoff, W.L. (1998). An improved Biotic Index of Organic stream pollution. Great Lakes Entomologist Journal, 20, 31-39.

Leiva, M. (2004). Macroinvertebrados bentónicos como bioindicadores de la calidad de agua en la cuenca del Estero Peru comuna de Lautaro. [Tesis de licenciatura en recursos naturales] Universidad católica de Toluca.

Roldan, G. (1997). Los macroinvertebrados como indicadores de la calidad del agua, en bioindicadores ambientales de la calidad del agua. Universidad del Valle. 
Roldan, G. (1999). Los macroinvertebrados y su valor como indicadores de la calidad del agua. Rev Acad Colomb Cienc, 23(88),375-387.

Romero, R. (2011). Clave dicotómica para la identificación de macroinvertebrados de la cuenca del Ebro. Confederación hidrográfica del Ebro.

Stroud Water Research Center. (2012). Leaf Pack Network. Stroud Water Research Center Zamorra, H. (2011). El índice BWMP y la evaluación biológica de la calidad del agua en los ecosistemas acuáticos epicontinentales naturales de Colombia. Universidad del Cauca.

\section{Anexo 1.}

ORDEN EPHEMEROPTERA

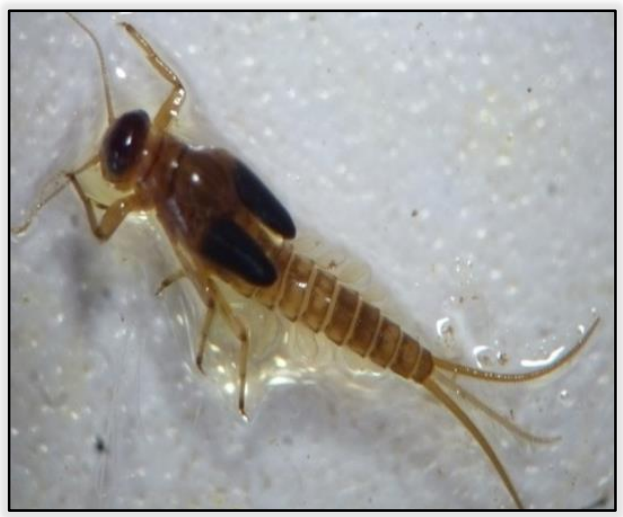

Familia: Baetidae

\section{ORDEN PLECOPTERA}

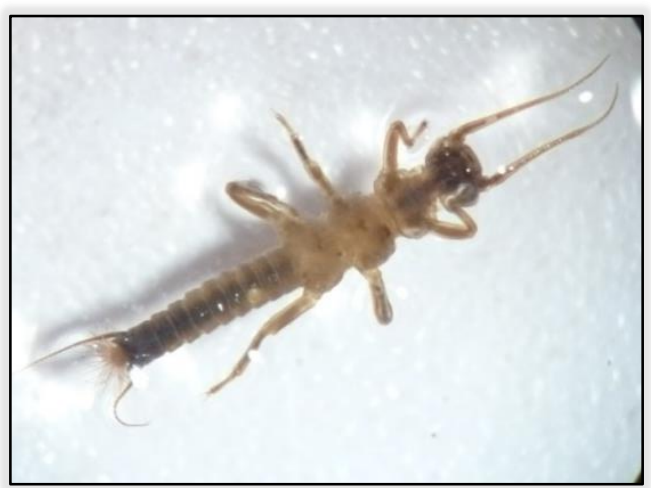

Familia: Gripopterygidae
ORDEN TRICHOPTERA

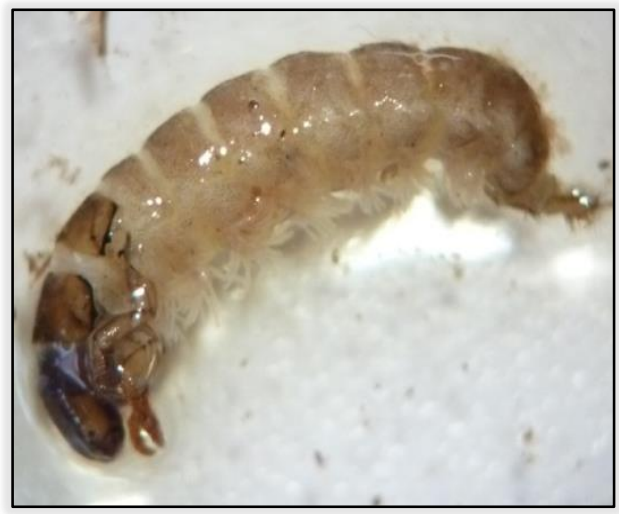

Familia: Hydropsychidae

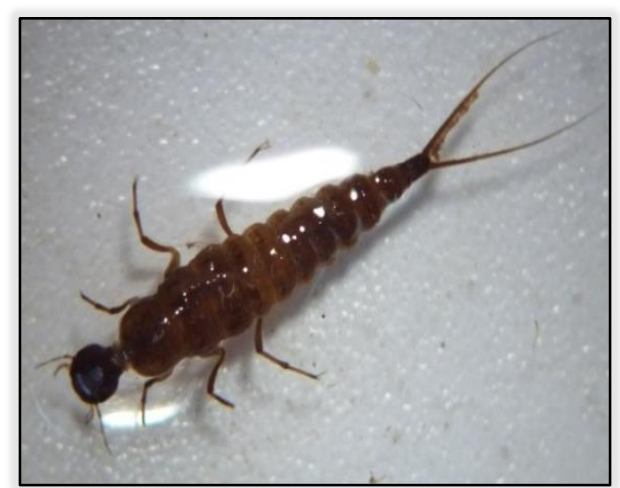

Familia: Gerridae 
ORDEN ODONATA

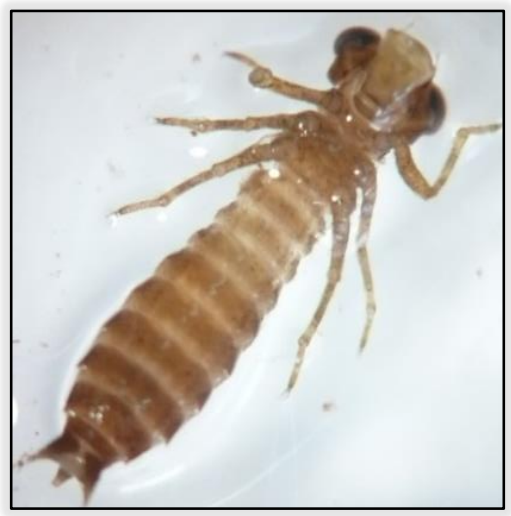

Familia: Aeshnidae

ORDEN HETEROPTERA

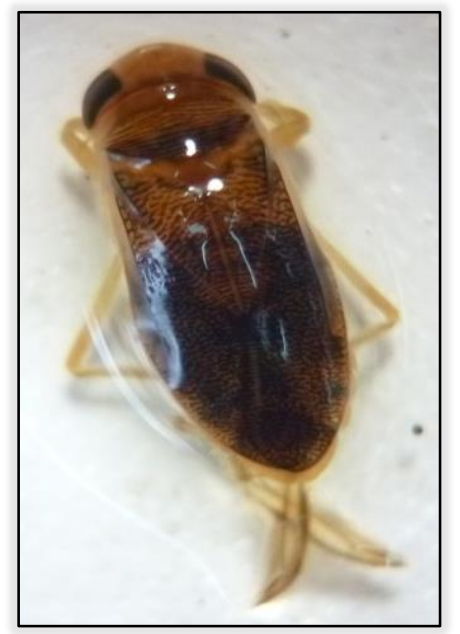

Familia: Notonectidae

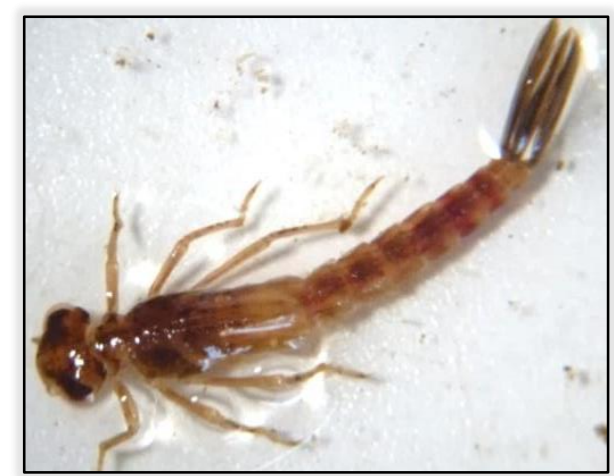

Familia: Coenagrionidae ORDEN AMPHIPODA

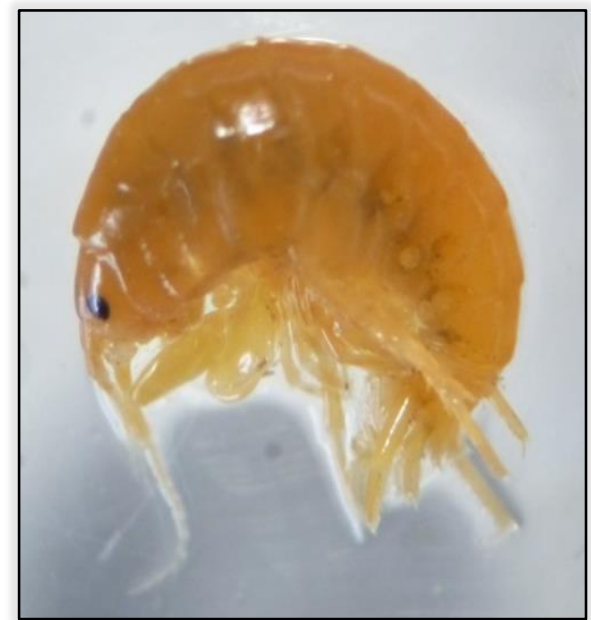

Familia: Hyalellidae 